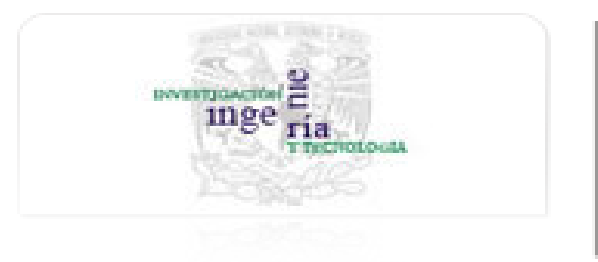

Ingeniería. Investigación y Tecnología

ISSN: 1405-7743

iit.revista@gmail.com

Universidad Nacional Autónoma de México

México

Campos-Aranda, D.F.

Identificación del número N mediante el método del HUT, en siete cuencas del alto río Grijalva, México Ingeniería. Investigación y Tecnología, vol. XII, núm. 3, septiembre, 2011, pp. 269-276

Universidad Nacional Autónoma de México

Distrito Federal, México

Disponible en: http://www.redalyc.org/articulo.oa?id=40420773003

Cómo citar el artículo

- Número completo

- Más información del artículo

Página de la revista en redalyc.org

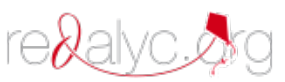

Sistema de Información Científica

Red de Revistas Científicas de América Latina, el Caribe, España y Portugal

Proyecto académico sin fines de lucro, desarrollado bajo la iniciativa de acceso abierto 


\title{
Identificación del número $N$ mediante el método del HUT, en siete cuencas del alto río Grijalva, México
}

\author{
N Number Identification Through the TUH Method, \\ in Seven Watersheds of the Upper Grijalva River, in Mexico
}

\author{
Campos-Aranda D.F. \\ Facultad de Ingeniería \\ Universidad Autónoma de San Luis Potosí \\ E-mail:campos_aranda@hotmail.com
}

\section{Información del artículo: recibido: abril de 2008, aceptado: agosto de 2010}

\section{Resumen}

Se destaca la gran complejidad involucrada en los procesos hidrológicos que generan las crecientes. Por ello, la identificación o calibración de cualquier método de predicción de crecientes, será siempre una acción que ayudará a su estimación, más confiable, en la cual influye el parámetro que se calibra. Primero, se describe brevemente el método del hidrograma unitario triangular (HUT) y con detalle la estimación de su tormenta de diseño con base en la fórmula de Chen, así como la estrategia necesaria para la identificación del número $N$, asociado a cada uno de los seis periodos de retorno procesados. La calibración se realizó en siete cuencas rurales de la Región Hidrológica Núm. 30 Parcial (alto río Grijalva), cuyas áreas de cuenca variaron de 190 a $4,768 \mathrm{~km}^{2}$. Posteriormente, se citan los diferentes factores que influyen en los resultados. Por último, se formulan conclusiones que destacan la importancia de los resultados numéricos de este estudio y de su aplicación sistemática regional durante la estimación de crecientes en cuencas sin aforos.

\footnotetext{
Abstract

The complexity of the hydrological processes involved in flood generation is pointed out. Hence, the calibration or identification of any flood prediction method will always be an action to help build a more accurate estimation. First, the triangular unit hydrograph (TUH) method is described briefly, the design storm estimation with base in the Chen formula and the necessary strategy for $N$ number identification are described in detail, associated to each one of the six recurrence intervals processed. The calibration was made in seven rural watersheds of the Hydrological Region Núm. 30 Partial (upper Grijalva river), with watershed areas varying from 190 to $4,768 \mathrm{~km}^{2}$. Next, the different factors which affected the results are listed. Lastly,
}

\section{Descriptores}

- hidrograma unitario triangular

- fórmula de Chen

- predicciones de lluvia diaria

- predicciones de gastos máximos

\section{Keywords}

- triangular unit hydrograph

- Chen formula

- predictions of daily rainfall

- predictions of maximum flows 
conclusions are formulated pointing out the importance of the numerical results of this study and their regional systematic application in the flood estimation processes in watershed without hydrometric data.

\section{Introducción}

La estimación de crecientes de diseño constituye la parte fundamental de un estudio hidrológico encaminado a dimensionar diferentes obras de infraestructura hidráulica, tales como: alcantarillas y puentes carreteros y de ferrocarril, altura de bordos o diques de protección de ciudades y áreas importantes relacionadas con la economía del lugar, así como los vertedores de todo tipo de presas de aprovechamiento y/o control.

Por otra parte, el sistema hidrológico que produce las crecientes, es decir, la interacción de la atmósfera con la geomorfología de la cuenca, los suelos y vegetación de ésta, así como las actividades del hombre, es tan complejo que su modelación únicamente puede proporcionar estimaciones aproximadas, mismas que estarán en correspondencia con la calidad de la información utilizada. De manera general, en todo sistema hidrológico se conjugan o interrelacionan la entrada, la operación del sistema y su salida, esto es (Dooge, 1973):

$y(t)=h(t) \psi x(t)$

donde $\psi$ es un símbolo que indica que la función de operación $h(t)$ y la función de entrada $x(t)$ se combinan de alguna forma para producir la función de salida $y(t)$. Cuando se conocen dos de las tres funciones citadas, es posible obtener la desconocida; cuando ésta es la salida el problema es de predicción, cuando la función buscada es la operación del sistema se llama identificación y cuando es la entrada el problema se conoce como detección.

La enorme complejidad involucrada en la génesis de las crecientes origina que la mejor información que se puede obtener para realizar estimaciones de éstas, es la que procede de los registros sistemáticos e históricos de las avenidas que han ocurrido en el pasado. Entonces, la utilización de la información hidrométrica local sobre crecientes, para la calibración (identificación) de cualquier modelo o método de estimación, es uno de los enfoques más confiables y seguros para lograr estimaciones apegadas a la realidad.

Para la estimación de crecientes en cuencas rurales se han propuesto docenas de fórmulas empíricas y un gran número de métodos basados en la relación lluviaescurrimiento, también denominados métodos hidrológicos. Uno de los más confiables y difundidos, es el conocido como método del hidrograma unitario trian- gular (HUT), el cual se puede consultar, entre otros, en Miller y Clark (1966), Miller et al. (1977) y Campos (1982, 1991).

El objetivo fundamental de este trabajo consistió en la identificación del número $N$ de la curva de escurrimiento, obtenido al igualar las predicciones de siete cuencas rurales aforadas de la Región Hidrológica Núm. 30 Parcial (alto río Grijalva), con las obtenidas a través del método del HUT, aplicando una aproximación empírica al tiempo de concentración (Tc) de cada cuenca y tormentas de diseño estimadas con la fórmula de Chen, la cual se basa en la información pluviográfica y pluviométrica disponibles en tal región. Además, se mencionan los factores que influyen en el proceso de calibración del número $\mathrm{N}$ y se formulan las correspondientes conclusiones, en las cuales se destaca la importancia de los resultados numéricos y de la aplicación sistemática de este proceso en otras regiones del país y en las estimaciones de crecientes en cuencas rurales sin aforos.

La obtención del número $N$ con base en datos de lluvia y su respectivo escurrimiento comenzó en la década de los años ochenta, como describen exhaustivamente Mishra et al. (2008). Más recientemente, la aplicación de los sistemas de información geográfica ha permitido la estimación del número $N$ en cuencas a partir de la información detallada de su cobertura vegetal, como lo han expuesto Miranda et al. (2009).

\section{Desarrollo}

\section{Método del HUT}

El hidrograma unitario (HU) se define como el hidrograma de gasto resultante de una lluvia en exceso unitaria ocurriendo uniformemente sobre la cuenca y durante la duración unitaria especificada (WMO, 1974). El método del hidrograma unitario triangular (HUT) fue propuesto y utilizado en los Estados Unidos por el Bureau of Reclamation; en México se difundió desde 1966 como consecuencia de la traducción que se hizo del manual Design Small Dams, publicado originalmente en 1960.

En términos generales, el método del HUT consiste en establecer una tormenta de diseño con seis incrementos horarios, uno de 12 horas y otro final de 24 horas; se obtienen las precipitaciones en exceso $(\mathrm{Pe})$ correspondientes, respetando una pérdida mínima por infiltración, según sea el grupo hidrológico de suelos de 
la cuenca. En seguida se aplican tres HUT, uno para los incrementos horarios y los otros dos para los incrementos finales. Estos HUT son función del tiempo de concentración $(T c)$ de la cuenca, definido como el lapso desde que comenzó la lluvia en exceso hasta que todas las porciones de la cuenca de drenaje están contribuyendo simultáneamente al gasto en la salida (Pilgrim y Cordery, 1993). Por último, se suman las ordenadas en el inicio, pico y final de cada uno de los HUT parciales, para definir el hidrograma total buscado.

Las Pe se estiman con la fórmula del U. S. Soil Conservation Service, la cual es función del número $N$ de la curva de escurrimiento y de la precipitación acumulada (P), ésta es (Mockus, 1972):

$$
P e=\frac{\left(P-\frac{5,080}{N}+50.8\right)^{2}}{\left(P+\frac{20,320}{N}-203.2\right)}
$$

\section{Cuencas procesadas}

Las cuencas estudiadas corresponden a siete de las nueve que procesó Guichard (1998), las cuales están ubicadas en el área que drena hasta la presa Netzahualcóyotl (Malpaso) y que se conoce como cuenca del alto río Grijalva. Se eliminó la que corresponde a Puente Concordia, debido a que su área drenada está prácticamente en Guatemala y por lo tanto no se dispone de información pluviográfica ni pluviométrica en ella. Puente Colgante no se utilizó debido a que su régimen está afectado por la Presa Belisario Domínguez (La Angostura) y drena una cuenca demasiado grande de 24,765 $\mathrm{km}^{2}$. Las siete cuencas procesadas fueron: Yamonhó, Santo Domingo, Boquerón, Santa María, Río Blanco, Las Flores y San Miguel, cuyas características físicas proceden de los datos utilizados por Guichard (1998) y se presentan en la tabla 1. Actualmente, el uso de los modelos digitales de elevación y su procesa-

Tabla 1. Características físicas de las cuencas procesadas y tiempo de concentración $\left(T_{C}\right)$ estimado

\begin{tabular}{lclrrrrr}
\hline \multicolumn{1}{c}{ Nombre: } & \multicolumn{1}{c}{$\begin{array}{c}A \\
\text { Clave }^{*}\end{array}$} & \multicolumn{1}{c}{ Río aforado* } & $\begin{array}{c}\text { Lc } \\
\left(\mathrm{km}^{2}\right)\end{array}$ & $\begin{array}{r}\text { Sc } \\
(\mathrm{km})\end{array}$ & $\begin{array}{r}\text { H } \\
(\mathrm{adim})\end{array}$ & $\begin{array}{r}\text { Tc } \\
(\mathrm{m})\end{array}$ & $\begin{array}{r}\text { (h) } \\
(\mathrm{h})\end{array}$ \\
\hline 1. Yamonhó & $07-93$ & Yamonhó & 190.3 & 30 & 0.0286 & 1,630 & 3.0 \\
2. S. Domingo & - & Santo Domingo & $1,415.7$ & 81 & 0.0069 & 1,062 & 12.0 \\
3. Boquerón & $07-18$ & Suchiapa & $1,471.8$ & 88 & 0.0082 & 1.371 & 12.0 \\
4. Santa María & $07-81$ & Encajonado & $1,584.6$ & 80 & 0.0130 & 1,976 & 10.0 \\
5. Río Blanco & $07-69$ & Blanco & $1,878.8$ & 60 & 0.0107 & 1,220 & 8.0 \\
6. Las Flores & $07-43$ & Zoyatenco & $2,404.5$ & 95 & 0.0042 & 758 & 15.0 \\
7. San Miguel & - & San Miguel & $4,767.5$ & 160 & 0.0090 & 2,736 & 18.0 \\
\hline
\end{tabular}

miento permite la estimación de las propiedades físicas mostradas en la tabla 1, como lo han detallado Martínez y Campos (2010).

En la figura 1 se muestra la hidrografía general de la cuenca alta del río Grijalva y la ubicación de cada una de las siete cuencas procesadas, así como sus parteaguas. Esta figura se elaboró con base en las cartas del INEGI $(1981,1988)$ y SARH (1987).

\section{Tiempo de concentración $\left(T_{C}\right)$}

Para la estimación del Tc se utilizó una recopilación de fórmulas empíricas para cuencas rurales expuesta por Campos (2007), algunas de las cuales requieren el desnivel total $(H)$ en metros del colector principal. Para obtener una estimación de tal cantidad, no reportada por Guichard (1998), se multiplicó por un factor correctivo $(\mathrm{Fc})$ el desnivel que origina la pendiente promedio del cauce $(S c)$ al ser aplicada a su longitud $(L c)$, esto es:

$$
H=\mathrm{F} c \cdot(1,000 \cdot L c) \cdot S c
$$

El valor de Fc se adoptó igual 1.90, el cual corresponde al promedio de los encontrados por Martínez (2000) y Campos (2007). Los valores estimados con la ecuación 3 se tienen en la columna 7 de la tabla 1 y las magnitudes adoptadas para el Tc se exponen en la columna 8.

\section{Predicciones de gastos máximos}

Las siete series de gastos máximos anuales, correspondientes a las estaciones hidrométricas que serán procesadas, fueron tomadas de Guichard (1998) y se muestran en la tabla 2. Ya que las predicciones necesarias corresponden a periodos de retorno relativamente bajos $(\leq$ 100 años), es de esperarse que éstas resulten muy semejantes, por ello se adoptaron los valores medianos obtenidos a través de la aplicación de cinco criterios del análisis probabilístico, éstos fueron: las transformaciones MIMEMA y potencial, así como las distribuciones Log-Normal, Log-Pearson tipo III y GVE. Estos procedimientos se pueden consultar en Campos (2006). En cada periodo de retorno se eliminan sus

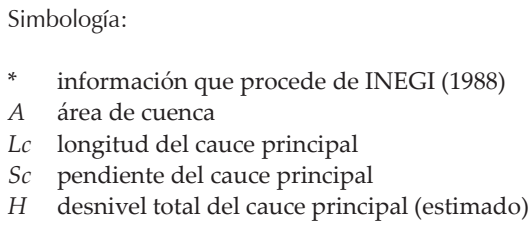




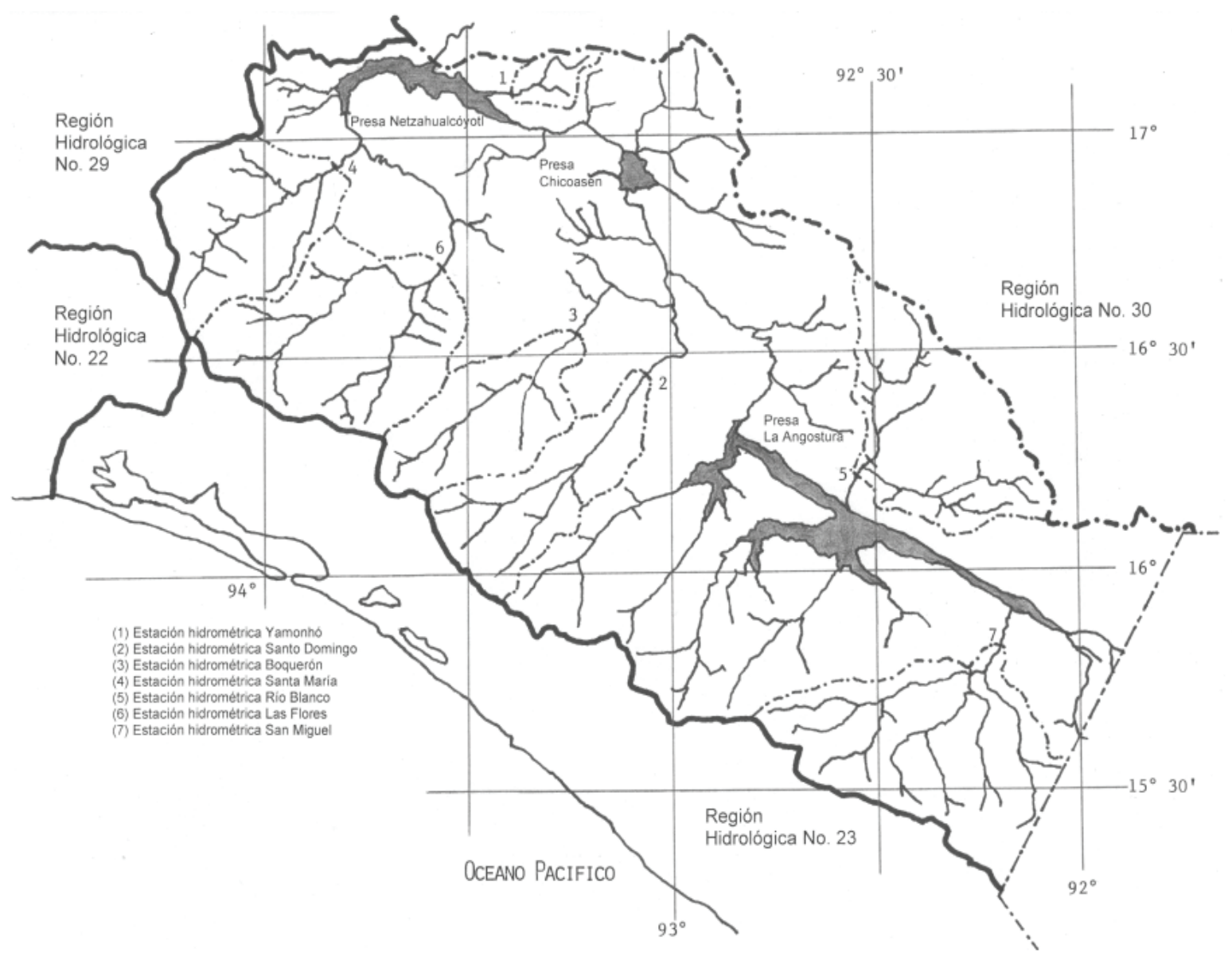

Figura 1. Locallización geográfica de las siete cuencas procesadas del alto río Grijalva

valores extremos (máximo y mínimo) dos veces y con ello se obtiene el valor mediano buscado.

En la tabla 3 se muestran los resultados de los cinco métodos probabilísticos aplicados a la primera y última estaciones hidrométricas procesadas, es decir, Yamonhó y San Miguel; en el resto únicamente se indican los valores mediados obtenidos.

\section{Lluvias de diseño}

El método del HUT requiere para su tormenta de diseño, las precipitaciones horarias durante las primeras seis horas y las de 12 y 24 horas de duración, asociadas al periodo de retorno que se analiza. Tales lluvias se estimaron con base en la fórmula de Chen (1983), aplicada según el procedimiento siguiente.

Paso 1. Se recaban en los mapas de isoyetas (SCT, 1990), para los centros de gravedad de las cuencas procesadas, las intensidades con duración de 60 minutos y periodos de retorno de 10, 25 y 50 años, que son los disponibles y que se designan por: $P_{1}^{10}, P_{1}^{25}, P_{1}^{50}$, pues equivalen a láminas de lluvia en milímetros, estos datos se presentan en la tabla 4 .

Paso 2. Se obtienen de las figuras A1.3 a A1.6 de Guichard (1998), denominadas isolíneas de precipitación puntual, las predicciones de lluvia diaria $\left(P_{D}^{T r}\right)$ de periodos de retorno 10, 25, 50 y 100 años, también para los centros de gravedad de las cuencas procesadas. Los valores de $P_{D}^{T r}$ se presentan en la tabla 4 , mismos que se multiplican por 1.13 (Weiss, 1964), para convertirlos en $P_{24}^{T r}$, con las cuales se obtienen los cocientes lluviaduración $(R)$ y lluvia-frecuencia $(F)$ necesarios para aplicar la fórmula de Chen, estos son:

$R=\frac{P_{1}^{T r}}{P_{24}^{T r}}$

$F=\frac{P_{24}^{100}}{P_{24}^{10}}$ 


\begin{tabular}{|c|c|c|c|c|c|c|c|}
\hline & & Santo & & Santa & Río & Las & San \\
\hline Año & Yamonhó & Domingo & Boquerón & María & Blanco & Flores & Miguel \\
\hline 1952 & & & 282 & & & & \\
\hline 1953 & & 177 & 181 & & & & \\
\hline 1954 & & 486 & 260 & & & & \\
\hline 1955 & & 610 & 272 & & & & \\
\hline 1956 & & 278 & 222 & & & & \\
\hline 1957 & & 246 & 80 & & & & \\
\hline 1958 & & 255 & 299 & & & & \\
\hline 1959 & & 295 & 168 & & & & \\
\hline 1960 & & 353 & 239 & & & & \\
\hline 1961 & & 300 & 139 & 651 & & & \\
\hline 1962 & & 265 & 290 & 284 & & 482 & \\
\hline 1963 & & 1740 & 623 & 559 & 152 & 798 & \\
\hline 1964 & & 675 & 158 & 365 & 173 & 436 & \\
\hline 1965 & & 625 & 130 & 244 & 107 & 243 & \\
\hline 1966 & & 898 & 238 & 440 & 89 & 309 & \\
\hline 1967 & & 475 & 185 & 409 & 47 & 272 & \\
\hline 1968 & 245 & 665 & 181 & 347 & 121 & 279 & \\
\hline 1969 & 269 & 587 & 296 & 529 & 224 & 349 & \\
\hline 1970 & 333 & & 323 & 1397 & 298 & 741 & \\
\hline 1971 & 39 & & 268 & 671 & 130 & 434 & \\
\hline 1972 & 160 & & 267 & 700 & 76 & 185 & \\
\hline 1973 & 203 & & 501 & 1453 & 329 & 1163 & \\
\hline 1974 & 354 & & & 638 & 111 & 379 & \\
\hline 1975 & 300 & & & 432 & 98 & 192 & \\
\hline 1976 & 86 & 88 & & 387 & 125 & 142 & 1069 \\
\hline 1977 & 107 & 118 & & 401 & 117 & 80 & 1016 \\
\hline 1978 & 372 & 276 & & 537 & 165 & 311 & 1250 \\
\hline 1979 & 632 & 280 & & 500 & 213 & 302 & 1566 \\
\hline 1980 & 129 & 1418 & & 3247 & 139 & 9350 & 1670 \\
\hline 1981 & 220 & 256 & & 1060 & 173 & 581 & 1552 \\
\hline 1982 & 131 & 287 & & 345 & 133 & 154 & 1053 \\
\hline 1983 & 79 & 204 & & 396 & 175 & 499 & 934 \\
\hline 1984 & 148 & 325 & & 1008 & & & 1199 \\
\hline 1985 & 298 & 191 & 166 & 291 & & 190 & 942 \\
\hline 1986 & 621 & 141 & 306 & & & & 590 \\
\hline 1987 & 111 & 147 & 173 & & & & 1166 \\
\hline 1988 & 363 & 474 & 271 & & & 1881 & 1396 \\
\hline 1989 & 517 & 534 & 768 & & & 2891 & 1667 \\
\hline 1990 & & 187 & 100 & & & 119 & 734 \\
\hline 1991 & & 41 & 112 & 220 & & 163 & 1054 \\
\hline 1992 & & 276 & 184 & 252 & & 184 & 1589 \\
\hline 1993 & & 200 & 260 & & & 258 & 1408 \\
\hline 1994 & 297 & 35 & 73 & 303 & & 64 & 859 \\
\hline 1995 & 355 & 153 & & 454 & & & 1440 \\
\hline 1996 & 106 & 111 & & 277 & & & 1013 \\
\hline
\end{tabular}

\begin{tabular}{lcrrrrrr}
\hline \multicolumn{1}{c}{ Estación hidrométrica } & EEA & \multicolumn{7}{c}{ Periodos de retorno en años } \\
\multicolumn{1}{c}{ Método de ajuste aplicado } & $\left(\mathrm{m}^{3} / \mathrm{s}\right)$ & 2 & 5 & 10 & 25 & 50 & 100 \\
\hline 1. Yamonhó & & & & & & & \\
Transformación MIMEMA & 36.8 & 226 & 389 & $\mathbf{4 8 3}$ & 584 & 649 & 707 \\
Transformación Potencial & 36.2 & $\mathbf{2 2 6}$ & $\mathbf{3 7 9}$ & 482 & $\mathbf{6 1 2}$ & $\mathbf{7 0 7}$ & $\mathbf{8 0 2}$ \\
Distribución Log-Normal (2p) & 34.6 & 210 & 373 & 504 & 694 & 854 & 1028 \\
Distribución Log-Pearson tipo III & 32.4 & 225 & 381 & 486 & 613 & 703 & 789 \\
Distribución GVE & 33.3 & 231 & 379 & 480 & 611 & 710 & 810 \\
2. Santo Domingo & - & $\mathbf{2 8 3}$ & $\mathbf{5 6 5}$ & $\mathbf{7 9 4}$ & $\mathbf{1 1 2 2}$ & $\mathbf{1 4 2 3}$ & $\mathbf{1 6 8 5}$ \\
3. Boquerón & - & $\mathbf{2 1 7}$ & $\mathbf{3 3 8}$ & $\mathbf{4 2 8}$ & $\mathbf{5 5 4}$ & $\mathbf{6 5 6}$ & $\mathbf{7 6 5}$ \\
4. Santa María & - & $\mathbf{4 3 7}$ & $\mathbf{7 5 2}$ & $\mathbf{1 1 1 0}$ & $\mathbf{1 8 6 7}$ & $\mathbf{2 6 5 1}$ & $\mathbf{3 8 4 0}$ \\
5. Río Blanco & - & $\mathbf{1 3 9}$ & $\mathbf{2 0 2}$ & $\mathbf{2 4 2}$ & $\mathbf{2 9 5}$ & $\mathbf{3 3 3}$ & $\mathbf{3 7 0}$ \\
6. Las Flores & - & $\mathbf{3 0 7}$ & $\mathbf{8 5 2}$ & $\mathbf{1 4 2 2}$ & $\mathbf{3 3 3 4}$ & $\mathbf{5 6 2 4}$ & $\mathbf{7 2 7 8}$ \\
7. San Miguel & & & & & & & \\
Transformación MIMEMA & 607.5 & 1133 & 2092 & 2886 & 3733 & 4281 & 4773 \\
Transformación Potencial & 735.3 & $\mathbf{1 1 9 6}$ & 1610 & 1990 & 2708 & 3607 & 5298 \\
Distribución Log-Normal (2p) & 723.0 & 1287 & 1803 & 2150 & 2593 & 2928 & 3265 \\
Distribución Log-Pearson tipo III & 617.3 & 1175 & 1968 & 2621 & 3602 & 4453 & 5413 \\
Distribución GVE & 632.4 & 1197 & $\mathbf{1 9 4 2}$ & $\mathbf{2 5 4 1}$ & $\mathbf{3 4 4 1}$ & $\mathbf{4 2 3 3}$ & $\mathbf{5 1 4 1}$ \\
\hline
\end{tabular}

Tabla 2. Gastos máximos anuales $\left(\mathrm{m}^{3} / \mathrm{s}\right)$ en siete estaciones hidrométricas de la cuenca del alto río Grijalva
Tabla 3. Predicciones estimadas $\left(\mathrm{m}^{3} / \mathrm{s}\right)$ en las siete estaciones hidrométricas indicadas de la cuenca del alto Río Grijalba 


\begin{tabular}{lccccccccc}
\hline \multicolumn{1}{c}{ Estación } & \multicolumn{2}{c}{ Centro de gravedad } & & & & & & \\
\multicolumn{1}{c}{ hidrométrica } & Latitud & Longitud & $P_{1}^{10}$ & $P_{1}^{25}$ & $P_{1}^{50}$ & \multicolumn{1}{c}{$P_{D}^{10}$} & $P_{D}^{25}$ & $P_{D}^{50}$ & $P_{D}^{100}$ \\
\hline 1. Yamonhó & $17^{\circ} 10^{\prime}$ & $93^{\circ} 15^{\prime}$ & 75 & 90 & 105 & 185 & 220 & 250 & 300 \\
2. Santo Domingo & $16^{\circ} 12^{\prime}$ & $93^{\circ} 20^{\prime}$ & 70 & 85 & 110 & 114 & 140 & 150 & 185 \\
3. Boquerón & $16^{\circ} 22^{\prime}$ & $93^{\circ} 28^{\prime}$ & 60 & 76 & 96 & 90 & 120 & 125 & 150 \\
4. Santa María & $16^{\circ} 45^{\prime}$ & $94^{\circ} 02^{\prime}$ & 73 & 78 & 90 & 138 & 170 & 190 & 210 \\
5. Río Blanco & $16^{\circ} 10^{\prime}$ & $92^{\circ} 12^{\prime}$ & 65 & 81 & 85 & 90 & 125 & 130 & 150 \\
6. Las Flores & $16^{\circ} 31^{\prime}$ & $93^{\circ} 45^{\prime}$ & 71 & 78 & 94 & 112 & 130 & 145 & 160 \\
7. San Miguel & $15^{\circ} 30^{\prime}$ & $92^{\circ} 10^{\prime}$ & 76 & 82 & 98 & 100 & 120 & 130 & 140 \\
\hline
\end{tabular}

Tabla 4. Características estadísticas de la información pluviográfica y pluviométrica disponible en las siete cuencas procesadas del alto Río Grijalva
Paso 3. Con el valor promedio $\left(R_{\text {prom }}\right)$ de los tres cocientes $R$ que se pueden evaluar, uno para cada periodo de retorno de 10, 25 y 50 años, se obtienen los parámetros $a, b$ y $c$ de la fórmula de Chen, ecuación 12, con las expresiones propuestas por Campos (2007):

$$
\begin{aligned}
a= & -2.297536+100.0389 \cdot R-432.5438 \cdot R^{2}+1256.228 \cdot R^{3} \\
& -1028.902 \cdot R^{4} \\
b= & -9.845761+96.94864 \cdot R-341.4349 \cdot R^{2}+757.9172 \cdot R^{3} \\
& -598.7461 \cdot R^{4}
\end{aligned}
$$$$
c=-0.06498345+5.069294 \cdot R-16.08111 \cdot R^{2}+29.09596 \cdot R^{3}
$$$$
-20.06288 \cdot R^{4}
$$

Las fórmulas anteriores son aplicables únicamente en el intervalo $0.10 \leq R \leq 0.60$. Extrapolando las curvas originales de Chen hasta $R=0.70$, se definieron las expresiones siguientes válidas en el intervalo $0.20 \leq R \leq 0.70$ :

$$
\begin{aligned}
a= & 21.03453-186.4683 \cdot R+825.4915 \cdot R^{2}-1,084.846 \cdot R^{3}+ \\
& 524.06 \cdot R^{4}
\end{aligned}
$$

$$
\begin{aligned}
b= & 3.487775-68.13976 \cdot R+389.4625 \cdot R^{2}-612.4041 \cdot R^{3}+ \\
& 315.8721 \cdot R^{4}
\end{aligned}
$$

$c=0.2677553+0.9481759 \cdot R+2.109415 \cdot R^{2}-4.827012 \cdot R^{3}$

$$
+2.459584 \cdot R^{4}
$$

La fórmula de Chen es la siguiente:

$$
P_{t}^{T r}=\frac{a \cdot P_{1}^{10} \cdot \log \left(10^{2-F} \operatorname{Tr}^{F-1}\right) \cdot t}{60(t+b)^{c}}
$$

donde $P_{t}^{T r}$ y $P_{1}^{10}$ están en milímetros, $\mathrm{t}$ en minutos $(5 \leq \mathrm{t}$ $\leq 1,440)$ y $\operatorname{Tr}(5 \leq \operatorname{Tr} \leq 100)$ en años.

Paso 4. Las estimaciones de la fórmula de Chen son de tipo puntual y por ello se deben ajustar al tamaño de cada cuenca analizada, ya que todas las tormentas son menos intensas conforme abarcan más área. El factor de reducción $(F r)$ que se aplicó procede del USACE (1981) y se obtiene con la fórmula siguiente:

$$
F r=1.0-0.3549 \cdot D^{-0.42723}\left(1.0-e^{-0.005794 \cdot A}\right)
$$

en la cual, $D$ es la duración de la precipitación en horas

\begin{tabular}{|c|c|c|c|c|c|c|c|c|c|c|c|c|c|c|c|}
\hline \multirow[t]{2}{*}{ Nombre } & \multicolumn{5}{|c|}{ Parámetros de la fórmula de Chen } & \multirow[t]{2}{*}{$\operatorname{Tr}$} & \multirow[t]{2}{*}{2} & \multirow[t]{2}{*}{5} & 10 & 25 & 50 & 100 & $\mathrm{cN}$ & \multirow{2}{*}{$\begin{array}{l}\text { Valor } \\
\text { medio }\end{array}$} & \multirow{2}{*}{$\begin{array}{c}\text { Valor } \\
\text { mediano }\end{array}$} \\
\hline & $R$ & $F$ & $a$ & $b$ & $c$ & & & & \multicolumn{5}{|c|}{ Números $N$} & & \\
\hline 1. Yamonhó & 0.364 & 1.622 & 19.343 & 6.252 & 0.701 & & 70.7 & 64.2 & 59.1 & 52.5 & 48.2 & 44.9 & $\downarrow$ & - & - \\
\hline 2. Santo Domingo & 0.577 & 1.623 & 38.666 & 11.650 & 0.872 & & 81.9 & 78.7 & 76.6 & 74.4 & 73.8 & 72.3 & $\downarrow \approx$ & 76.3 & 75.5 \\
\hline 3. Boquerón & 0.610 & 1.667 & 40.778 & 11.573 & 0.876 & & 84.5 & 75.3 & 70.2 & 65.4 & 62.3 & 59.6 & $\downarrow$ & - & - \\
\hline 4. Santa María & 0.431 & 1.522 & 25.554 & 8.538 & 0.770 & & 69.8 & 67.2 & 66.3 & 68.2 & 71.1 & 77.3 & $\downarrow \uparrow \approx$ & 70.0 & 69.0 \\
\hline 5. Río Blanco & 0.597 & 1.667 & 39.863 & 11.551 & 0.872 & & 70.6 & 60.6 & 54.8 & 48.7 & 44.8 & 40.9 & $\downarrow$ & - & - \\
\hline 6. Las Flores & 0.555 & 1.429 & 37.142 & 11.552 & 0.866 & & 69.9 & 76.6 & 81.3 & - & - & - & $\uparrow$ & - & - \\
\hline 7. San Miguel & 0.648 & 1.400 & 44.060 & 11.932 & 0.888 & & 77.8 & 78.9 & 79.4 & 80.4 & 81.7 & 83.3 & $\uparrow \approx$ & 80.2 & 79.9 \\
\hline
\end{tabular}
y $A$ es el área de cuenca en $\mathrm{km}^{2}$.

\section{Proceso de identificación del número $N$}

En cada una de las siete cuencas analizadas, se tienen como datos básicos su área de cuenca, su tiempo de concentración y las estimaciones de su gasto máximo asociado a los seis periodos de retorno estudiados; además, se adoptó en todos los casos un suelo tipo B, para la consideración de pérdidas mínimas del método del HUT.

Tabla 5. Resultados de la fórmula de Chen y de la identificación del número $N$ con el método del HUT, en las siete cuencas indicadas del alto río Grijalba

Simbología:

$\begin{array}{llll}R & \text { cociente lluvia-duración } & \mathrm{cN} & \text { comportamiento del número } N \\ F & \text { cociente lluvia-frecuencia } & \downarrow & \text { disminuye conforme crece } \mathrm{el} \mathrm{Tr} \\ a, b, c & \text { parámetros función de } R & \uparrow & \text { aumenta conforme crece el } \mathrm{Tr} \\ \mathrm{Tr} & \text { periodo de retorno en años } & \approx & \text { muestra escasa fluctuación }\end{array}$


El proceso de calibración se realizó por prueba y error en cada cuenca, asignando un número $N$ para obtener una predicción a través del método del HUT, igual a la estimación de su gasto máximo observado en el periodo de retorno analizado. Los resultados numéricos de este proceso se resumen en la tabla 5, tanto para el número $N$ como para los parámetros calculados de la fórmula de Chen.

\section{Análisis y discusión de resultados}

Antes de comenzar la interpretación de los resultados numéricos (tabla 5), es conveniente cuando menos citar los factores más importantes que influyen en la calibración del número $N$. En primer término, están los factores asociados al propio método del HUT, como son la estructura de su tormenta de diseño, la simplificación geométrica del hidrograma unitario y la veracidad en la estimación de las lluvias en exceso a través de la ecuación 2. Dentro de la estimación de las lluvias de diseño, influyen:

a) el trazo de las curvas isoyetas de intensidades y de lluvia diaria,

b) la obtención de sus valores representativos en cada cuenca y

c) la representatividad de las tormentas a través de la fórmula de Chen y del factor de reducción adoptado. Incluso la estimación de las predicciones de gasto máximo observado pueden inducir fuertes dispersiones en el número $N$ calibrado, como fue el caso en la estación hidrométrica Las Flores.

Tomando en consideración la multitud de factores que determinan el calibrado del número $N$, se concluye que los resultados numéricos de la tabla 5 son bastante consistentes, ya que en tres de las siete cuencas procesadas se tiene poca variación, además estas cuencas (Santa María, Santo Domingo y San Miguel) presentan un incremento en el valor del número $N(70,76$ y 80) conforme su localización, ya que pasan de la parte baja hacia la porción alta, lo cual se aprecia en una disminución de la latitud y longitud de sus centros de gravedad (tabla 4).

Por otra parte, los datos de la tabla 2 permiten identificar a las estaciones Santo Domingo, Santa María, Las Flores y San Miguel como registros con presencia de valores dispersos (outliers). De tales series, la de Las Flores presenta un valor tan extremo que induce predicciones de tal magnitud que no se pueden igualar con el método del HUT, en los periodos de retorno $\geq 25$ años (ver tabla 5), debido a que las lluvias de diseño no siguen tal comportamiento y al hecho de tener que respetar las pérdidas mínimas por infiltración.

Los valores extremos calibrados para el número $N$ en la región estudiada, es decir, 84.5 como máximo y 40.9 como mínimo, dan testimonio de la poca exactitud de las estimaciones respecto a los valores reales o exactos, por ejemplo en los tiempos de concentración y las lluvias de diseño, lo cual se observa en las estaciones Yamonhó, Boquerón y Río Blanco.

\section{Conclusiones}

Los resultados globales de la identificación del número $N$, indican que en la Región Hidrológica Núm. 30 Parcial (alto río Grijalva), este parámetro tiene un valor representativo que oscila entre 70 y 80 , con su valor mínimo, medio (76) y máximo en las cuencas de las estaciones hidrométricas Santa María, Santo Domingo y San Miguel, respectivamente.

En general, el procedimiento de calibración descrito es aplicable en toda cuenca rural aforada que incluya estaciones pluviométricas dentro de ella, o como mínimo en su estación hidrométrica. Por ello, se recomienda utilizarlo en otro tipo de zonas geográficas del país, para contar con un indicador del orden de magnitud global del número $N$, como el encontrado en la zona estudiada.

Además, durante la estimación de crecientes de diseño en cuencas rurales o urbanas sin aforos, se considera sumamente útil la calibración expuesta, realizando ésta en las cuencas circunvecinas ubicadas dentro de la misma zona geográfica. Los resultados pueden ser extrapolados a las cuencas no aforadas, con base en su precipitación media anual.

\section{Referencias}

Campos A.D.F. Manual para la estimación de avenidas máximas en cuencas y presas pequeñas. Cuarta Sección, capítulo 4: Métodos hidrológicos. pp. 85-114. Dirección General de Obras Hidráulicas e Ingeniería Agrícola para el Desarrollo Rural, S.A.R.H. México. 1982. 225 pp.

Campos A.D.F. Programa para el método del hidrograma unitario triangular de estimación de crecientes. Memoria del XVII Congreso de la Academia Nacional de Ingeniería, A. C. 18 al 20 de Septiembre de 1991. Monterrey, México. pp. 109-113.

Campos A.D.F. Análisis probabilístico univariado de datos hidrológicos. Avances en Hidráulica 13. AMH-IMTA. México. 2006. $172 \mathrm{pp}$

Campos A.D.F. Estimación y aprovechamiento del escurrimiento. Capítulo 2, tema 4: Parámetros hidrológicos de la cuenca, pp. 41-50 y Anexo 6: Aspectos de seguridad hidrológica en em- 
balses. pp. 411-429. Edición del autor. San Luis Potosí, México. 2007.440 pp.

Chen C.I. Rainfall Intensity-Duration-Frequency Formulas. Journal of the Hydraulics Engineering, 109 (12). 1983. Pp. 1603-1621.

Dooge J.C.I. Linear Theory of Hydrologic Systems. Technical Bulletin No. 1468 of the Agricultural Research Service. pp. 3-43. USDA. Washington, 1973.

Guichard R.D. Regionalización de lluvias y escurrimientos en la cuenca del alto río Grijalva. Tesis (Maestría en ingeniería) (Aprovechamientos hidráulicos). México DF. División de Estudios de Posgrado de la Facultad de Ingeniería de la Universidad Nacional Autónoma de México. Octubre de 1998. 124 pp.

Instituto Mexicano de Tecnología del Agua (IMTA). Banco Nacional de Datos de Aguas Superficiales (BANDAS). 8 CD's. Comisión Nacional del Agua-Secretaría de Medio Ambiente y Recursos Naturales-IMTA. Jiutepec, Morelos. 2003.

Instituto Nacional de Estadística, Geografía en Informática (INEGI). MERIDA. Carta Hidrológica. Aguas Superficiales. Escala 1:1’000,000. Aguascalientes, Ags. 1a. impresión. 1981.

Instituto Nacional de Estadística, Geografía en Informática (INEGI). VILLAHERMOSA. Carta Hidrológica. Aguas Superficiales. Escala 1:1'000,000. Aguascalientes, Ags. 2a. impresión. 1988.

Martínez M.S. I. Introducción a la hidrología superficial. Capítulo 2: Geomorfología de la cuenca. Pp. 27-53. Textos Universitarios. Universidad Autónoma de Aguascalientes. Aguascalientes, Ags. 2000. 327 pp.

Martínez M.S. I. y Campos A.D.F. Algoritmos para estimar características físicas en cuencas rurales y su aplicación en el calibrado del método Racional, en la Región Hidrológica Núm. 12 parcial (Río Santiago). Agrociencia, 44 (4).2010. pp. 393-408.

Miller D.L. y Clark R.A. Estudio de avenidas. Capítulo 2, pp. 5395. En: Diseño de presas pequeñas. US. Department of the Interior. CECSA. México. 1966. 639 pp.
Miller D.L., Clark R.A. \& Schamach S. Flood Studies. Chapter III, pp. 37-95. En: Design Small Dams. US. Bureau of Reclamation. Washington,. Revised Reprint. 1977. 816 pp.

Miranda A.L., Ibáñez C.L.A., Valdez L.J.R. y Hernández de la R.P. Modelación hidrológica empírica del gasto de 100 años de periodo de retorno del Río Grande, Tlalchapa, Guerrero en dos escenarios de uso del suelo. Agrociencia, 43(4):333-344. 2009.

Mishra S.K., Suresh-Babu P., Singh V.P. SCS-CN Method. Chapter 7, pp. 277-330. En: Hydrology and Hydraulics, Vijay P. Singh (ed.). Water Resources Publications, LLC. Highlands Ranch, Colorado, 2008. 1080 p.

Pilgrim D.H. \& Cordery I. Flood Runoff. Chapter 9, pp. 9.1-9.42. En: Handbook of Hydrology.David R. Maidment (ed.). McGraw-Hill, Inc. Nueva York. 1993.

Secretaría de Agricultura y Recursos Hidráulicos (SARH). Carta Hidrográfica. Regiones Hidrológicas 23, 29 y 30. Dirección General de Administración y Control de Sistemas Hidrológicos. México. 1987.

Secretaría de Comunicaciones y Transportes (SCT). Isoyetas de Intensidad-Duración Frecuencia. República Mexicana. Subsecretaría de Infraestructura de la SCT. México, DF. 1990. 495 p.

Mockus V. Estimation of Direct Runoff from Storm Rainfall. Chapter 10, pp. 10.1-10.24. On: Section 4: HYDROLOGY in National Engineering Handbook. US. Soil Conservation Service. Washington, DC, USA. 1972.

US. Army Corps of Engineers (USACE). HEC-1 Flood Hydrograph Package. Chapter 3: Rainfall-runoff simulation, pp. 8-42. Hydrologic Engineering Center. Davis, California, USA. 1981. $192 \mathrm{p}$.

Weiss L.L. Ratio of True Fixed-Interval Maximum Rainfall. Journal of Hydraulics Division, 90 (No. HYI):77-82.1964.

World Meteorological Organization (WMO). Glosario Hidrológico Internacional. Publicación Núm. 385. Ginebra, Suiza. 1974. 393 pp.

\section{Semblanza del autor}

Daniel Francisco Campos-Aranda. Obtuvo el título de ingeniero civil en diciembre de 1972 en la entonces Escuela de Ingeniería de la Universidad Autónoma de San Luis Potosí. Durante el primer semestre de 1977, realizó en Madrid, España un diplomado en hidrología general y aplicada. Posteriormente, durante 1980-1981, llevó a cabo estudios de maestría en ingeniería en la especialidad de hidráulica en la División de Estudios de Posgrado de la Facultad de Ingeniería de la UNAM. En esta misma institución, inició (1984) y concluyó (1987) el doctorado en ingeniería con especialidad en aprovechamientos hidráulicos. Ha publicado artículos principalmente en revistas mexicanas de excelencia: 35 en Ingeniería Hidráulica en México, 12 en Agrociencia y 8 en Ingeniería. Investigación y Tecnología. En congresos internacionales y nacionales ha presentado 24 y 73 ponencias, respectivamente. Fue investigador nacional (nivel I: expediente 7273) desde el $1^{\circ}$ de julio de 1991 hasta el 31 de diciembre del 2007. Actualmente es profesor jubilado de la UASLP, desde el $1^{\circ}$ de febrero del 2003. En 2008 la AMH le otorgó el premio nacional "Francisco Torres H." a la práctica profesional de la hidráulica. 